\title{
Development of Expandable Self-Capacitive Proximity and Tactile Sensor Module
}

\author{
Satoshi Tsujia, ,, Rei Nakamura ${ }^{\mathrm{a}}$, Teruhiko Kohama ${ }^{\mathrm{a}}$ \\ aFukuoka University, 8-19-1 Nanakuma, Jonan-ku, Fukuoka 814-0180, Japan \\ *Corresponding Author: tsuji@fukuoka-u.ac.jp
}

\begin{abstract}
Recently, collaborative robot which works in a same place with people has become attractive. We have proposed a self-capacitance proximity and tactile sensor for the safety and workability of collaborative robot so far. The sensor on the robot can detect an object before and after contact. In this paper, we propose a sensor module of the self-capacitance proximity and tactile sensor. The advantage of this sensor module is that an expansion and an implementation of the sensor are easy. This sensor module on the robot can detect objects before contact, and can detect pressure and its position after contact. The proposed sensor module may be useful system on collaborative robots.
\end{abstract}

Keywords: sensor module, proximity sensor, tactile sensor, collaborative robot.

\section{Introduction}

Many of workspaces of industrial robots are separated from people by safety fences for safety. Recently, collaborative robot which works in a same place with people has become attractive. The collaborative robot needs various sensors for safety. Among them, tactile sensors that detect a touch and proximity sensors that detect an object without contact are important for safety. Several types of tactile skin sensors for robots have been developed ${ }^{(1)-(4)}$. Tactile skin sensor using optical elements to detect contact pressure has been proposed ${ }^{(1)}$. The skin sensor can detect three components of an applied force from contact. A tactile sensor using a conductive sheet has been proposed ${ }^{(2)}$. The sensor can detect a contact pressure and a center point, while low wiring and high response speed. Triangular modules capacitance skin sensors to detect pressure has a measurement IC in the sensor, and can be connected to other sensors to cover a large area of robot surface ${ }^{(3)}$. In addition, collaborative robots using joint torque sensors have been on sale ${ }^{(4)}$. However, these tactile sensors and the robots are difficult to detect an object before a contact, and avoiding an object before contact.

To improve safety, human collaborative robots (HCRs) need to avoid contact with objects using proximity skin sensors. Several types of proximity sensors for robots have been proposed ${ }^{(5-7)}$. A proximity sensor using optical elements has been proposed ${ }^{(5)}$. The proximity sensor can detect an object and its center point before contact, while low wiring and high response speed. A proximity sensor using capacitance measurement has been proposed ${ }^{(6)}$. In addition, a collaborative robot with capacitive proximity sensors to avoid contact has been on sale ${ }^{(7)}$. However, these proximity sensors cannot detect a contact condition.

Proximity and tactile sensors to detect an object both before and after contact have been proposed ${ }^{(8-10)}$. A robotic skin sensor using an optical element can detect an object before and after contact ${ }^{(8)}$. However, it may be difficult to separate a proximity state and a contact state because the sensor obtains mixed proximity and contact information. A tactile and proximity sensors using mutual capacitance measurement have been proposed to detect an object at

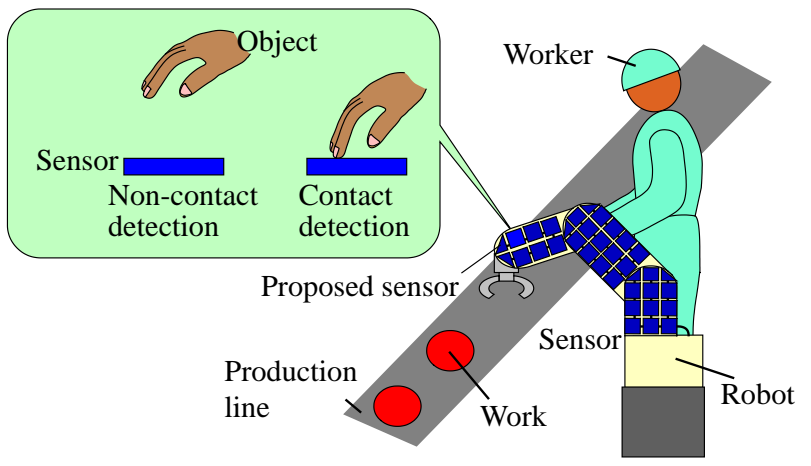

Fig. 1. Goal image of this study. 
proximity range and on a contact ${ }^{(9,10)}$. However, a mutual capacitance measurement requires binary electrodes, and the structural flexibility of the sensor is thereby degraded.

We focus on the tactile and proximity sensor for cooperative robots. Fig. 1 shows a goal image of the proposed sensor system on a collaborative robot that is working with humans. The sensor constantly measures the state near the robot surface, and uses that information to avoid contact with objects including humans. Thus, the skin sensor increases the workability and safety of HCRs.

We have developed a self-capacitance proximity and tactile sensor so far ${ }^{(11)}$. The self-capacitance measurement requires a single electrode. The sensor structure is simple because the sensor consists of two measurement electrodes and an elastic body. The measurement system is also simple because only self-capacitance measurement is used to the measurement. The sensor on a robot can detect objects both before and after contact. In addition, we have proposed an operating method for HCRs using the proposed self-capacitance proximity and tactile sensor ${ }^{(12)}$.

In this paper, we propose modularization of the proposed self-capacitance proximity and tactile sensor. The advantage of this sensor module is that an expansion and an implementation of the sensor are easy. Thus, it is possible to replace only the failed sensor to a new sensor when a sensor fails as shown in Fig. 1. The sensor module can be expected to speed up using the distributed process.

\section{Principle}

\subsection{Self-Capacitive Proximity and Tactile Sensor}

This proposed method ${ }^{(11)}$ uses only self-capacitance measurement for proximity and contact measurement. Fig. 2(a) shows a schematic diagram of the proposed sensor. The sensor consists of an upper electrode $\left(E_{1}\right)$, lower electrodes $\left(E_{2}\right)$, and an elastic body. A self-capacitance between $E_{1}$ or $E_{2}$ and ground is measured by switching between $E_{1}$ and $E_{2}$. Figs. 2(b) and (c) show an image of a proximity and contact measurement of the sensor. In Fig. 2(b), $\mathrm{E}_{1}$ is connected to a capacitance digital converter (CDC), a self-capacitance $\left(C_{2}\right)$ of $E_{1}$ is measured (Connection A). Here, $E_{2}$ is connected to a shield which is the same signal waveform of the measured electrode. In Fig. 2(c), $E_{2}$ is connected to $\mathrm{CDC}$, and a self-capacitance $\left(C_{1}\right)$ between $\mathrm{E}_{2}$ and $\mathrm{E}_{1}$ which is connected to ground, is measured (Connection B). In connection $\mathrm{A}, C_{2}$ change according to both a permittivity of the object and a distance between the sensor and the object, (a)

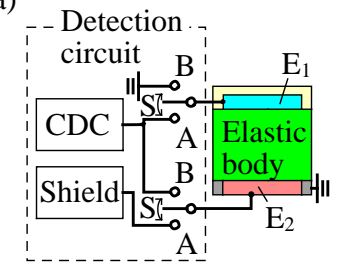

(b)

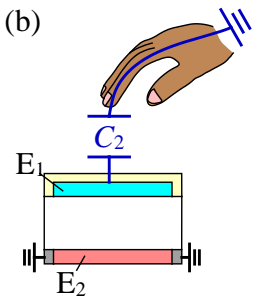

(c)

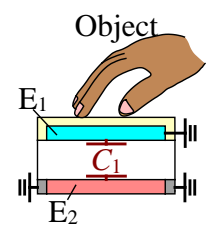

Fig. 2. Measurements by the proposed method ${ }^{(11)}$.

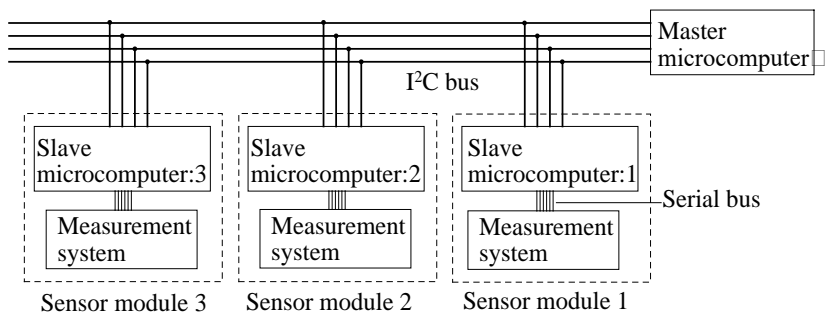

Fig. 3. Connection line of the proposed sensor module.

when the object approaches the sensor. Thus, the sensor can detect the object using $C_{2}$ before contact. Furthermore, the sensor can distinguish a material in the object using $C_{2}$ upon contact. In connection $\mathrm{B}, C_{1}$ do not change because $\mathrm{E}_{1}$ connected ground, when an object approaches the sensor. $C_{1}$ change according to an indentation which is equal to a distance between $E_{2}$ and $E_{1}$, when the object touches the sensor. Thus, the sensor can detect the contact condition using $C_{1}$. Therefore, the sensor can detect an object before and after contact using the self-capacitance measurement (11).

\subsection{Proximity and Tactile Sensor Module}

The self-capacitance proximity and tactile sensor module is used an $\mathrm{I}^{2} \mathrm{C}$ bus for communication between sensors (slaves) and microcomputer (master). The $\mathrm{I}^{2} \mathrm{C}$ bus is consisted of a serial data line (SDA), a serial clock line (SCL), a power supply line (Vdd), and a GND line. The address of each device connected to the $\mathrm{I}^{2} \mathrm{C}$ bus needs to be different. Thus, to connect devices with the same address on one $\mathrm{I}^{2} \mathrm{C}$ bus, the same number of SDA or SCL is required. However, SDA or SCL will increase and wiring will be complicated when the number of sensors is increased. Each sensor module has a slave microcomputers which has different device addresses as shown in Fig. 3. The slave microcomputers of each sensor communicate to the master microcomputer using one $\mathrm{I}^{2} \mathrm{C}$ bus. Each sensor module can be removed freely. Thus, it is possible to replace only the failed sensor to a new sensor when a sensor fails. 

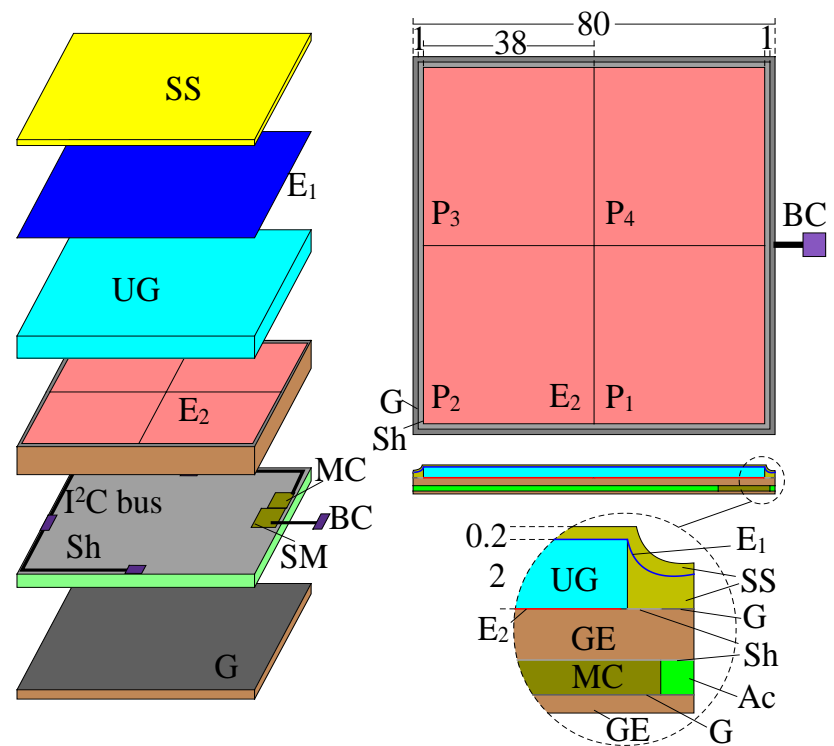

Fig. 4. Schematic diagram of the proposed sensor. $E_{1}$ and $\mathrm{E}_{2}$ : Electrodes, UG: Urethane gel, SS: Silicone sheet, Sh: Shield, G: ground, Ac: Acrylic, GE: Glass epoxy, MC: Measurement circuit, BC: Bus connector. (Dimensions in $\mathrm{mm})$.

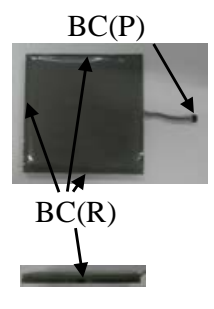

(a)

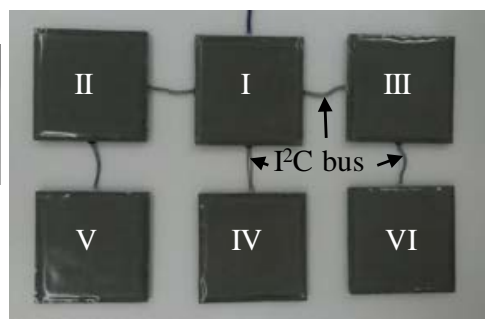

(b)

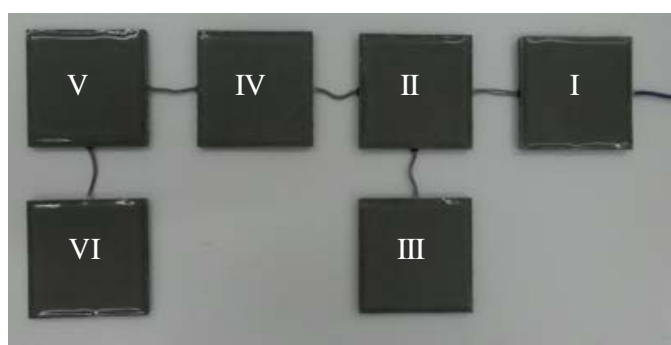

(c)
Fig. 5. Prototype sensor module.

\section{Prototype Sensor Module}

Figure 4 shows the prototype sensor module. In our design, $E_{1}(80 \times 80 \mathrm{~mm})$ is largely to increase sensitivity at a proximity range, and $\mathrm{E}_{2}(38 \times 38 \mathrm{~mm})$ is small to realize a high spatial resolution on contact. The size of the sensors is $80 \times 80 \mathrm{~mm}$. A silicone sheet (SS) was placed on top of the sensor to isolate the object and $E_{1}$. A urethane gel (UG) was placed between $E_{1}$ and $E_{2}$. To remove noise from the robot, a shield electrode (Sh) and GND electrode (G) were placed at the back of the sensor and around $E_{2}$. The self-capacitance measurement circuit (MC) consists of a capacitance measurement IC (Analog Devices, AD7147) and an analog switch for switching the $\mathrm{E}_{1}$ condition. A slave microcomputer (Microchip Technology, ATMEGA 168A, SM in Fig. 4) was used to control these and communicate to the master microcomputer. The $\mathrm{I}^{2} \mathrm{C}$ bus is used for communication between the slave microcomputer and the master microcomputer. Multiple sensor modules can be connected to one $\mathrm{I}^{2} \mathrm{C}$ bus line by setting different $\mathrm{I}^{2} \mathrm{C}$ addresses for each slave microcomputer. The sensor has a buffer circuit in consideration of connecting many sensor modules because the capacitance in $\mathrm{I}^{2} \mathrm{C}$ bus needs to $400 \mathrm{pF}$ or less. Fig. 5 shows the prototype sensor modules. Each sensor module can be connected by incorporating an $\mathrm{I}^{2} \mathrm{C}$ bus line and a bus connector (BC) in the sensor module. As shown in Fig. 5(a), each sensor has one plug connector (BC(P)) with cable and three receptacle connector (BC(R)) in the sensor. As shown in Figs. 5(b) and (c), setting position can be freely changed, and the sensor module can be easily added. It takes approximately $53 \mathrm{~ms}$ to obtain one complete cycle of measurements (six-sensor) with a data transmitting time from a microcomputer to PC.

\section{Results and Discussions}

\subsection{Basic Characteristics of the Sensor Module}

The proposed sensor system can detect an object both before and after contact ${ }^{(11)}$. We evaluated the proximity and contact measurements of the prototype sensor module. Objects are grounded conductors (GND), which are human model, and acrylic. The size of the object is $30 \times 30 \mathrm{~mm}$. The object is set on $\mathrm{P}_{1}$ of the sensor I. The distance between the sensor and the object is changed from 0 to $150 \mathrm{~mm}$ using the robot arm. The contact condition is measured using the force gauge. Fig. 6(a) shows $\Delta C_{2}$ of sensor I at proximity range, Fig. 6(b) shows the enlarged view of Fig. 6(a), and Fig. 6(c) shows $\Delta C_{1}$ of $\mathrm{P}_{1}$ on sensor I at proximity range. Here, $\Delta C_{1}$ and $\Delta C_{2}$ are the variation in $C_{1}$ and $C_{2}$. $\Delta C_{2}$ in Figs $6(\mathrm{a})$ and (b) changes according to the distance. Thus, the sensor can detect an object before contact using $\Delta C_{2}$. This sensor can detect an object (GND) within approximately $120 \mathrm{~mm}$. Here, proximity detection distance changes to an electrical characteristic of the object and an overlap between the sensor and the object. $\Delta C_{1}$ in Fig. 6(c) does not change at non-contact, and $\Delta C_{1}$ change after contact. Thus, the sensor can detect the contact using $\Delta C_{1}$ 


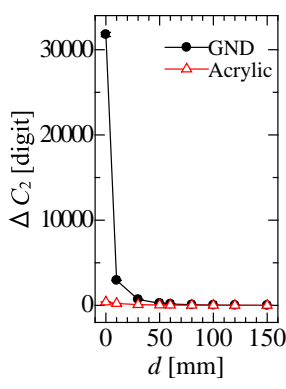

(a)

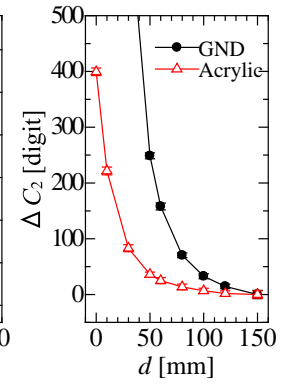

(b)

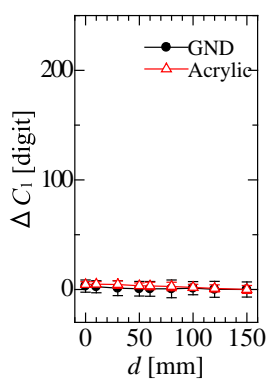

(c)
Fig. 6. Measurement results at proximity range.

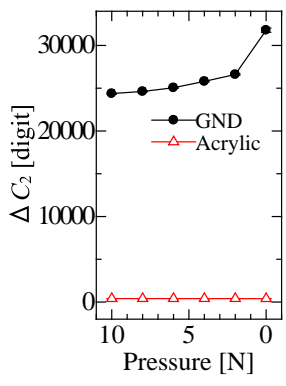

(a)

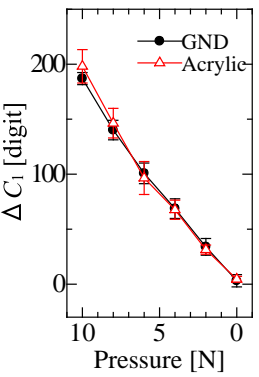

(b)

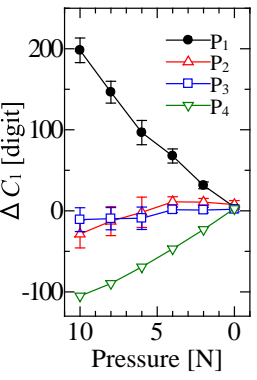

(c)
Fig. 7. Measurement results on contact.

(11). Fig. 7(a) shows $\Delta C_{2}$ on contact, Fig. 7(b) shows $\Delta C_{1}$ of $\mathrm{P}_{1}$ on contact, and Fig. 7(c) shows $\Delta C_{1}$ of each $\mathrm{E}_{2}$ on contact when the object is GND. $\Delta C_{2}$ in Fig. 7(a) change according to the permittivity of the object. Thus, the sensor can discriminate between the grounded conductor and other objects. $\Delta C_{1}$ in Fig. $7(\mathrm{~b})$ and (c) change according to pressure and its position. Thus, the sensor can detect approximate pressure and its position ${ }^{(11)}$.

\subsection{Proximity and Contact Measurement on the Robot Arm}

We evaluated the proximity and contact measurement using the prototype sensors, which were attached to the robot arm surface. Fig. 8 shows the prototype sensor on the robot arm. The sensor I to VI were connected as shown in Fig. 5(c). The object is the hand. Fig. 9 shows the experimental results. Fig. 9(a) shows the position between the sensor and the object (Hand). The hand is far away (A), the hand approached the robot (B to D). The finger pushed the sensor II with a weak force (E). Next, the finger pushed the sensor II with a strong force (F). Also, the finger pushed the sensor III (G). Fig. 9(b) shows the $\Delta C_{2}$, and Fig. 9(c) shows $\Delta C_{1}$. Here, $\Delta C_{2}$ and $\Delta C_{1}$ are denoted in white in Fig. 9 when the values are less than \pm 3 standard deviation (SD) lower than each value of air. In addition, $\Delta C_{2}$ in Fig. 9 shows 3000 digits when the values exceed 3000 digits, and $\Delta C_{1}$ in Fig. 9 shows 1000 digits when the values exceed

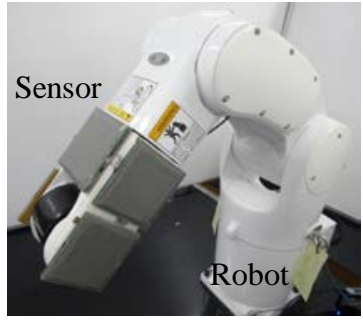

(a)

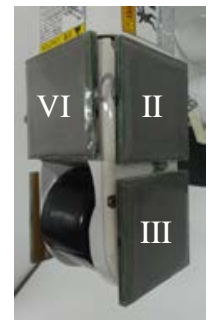

(b)

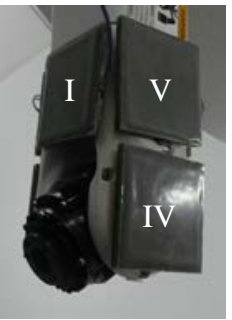

(c)
Fig. 8. Prototype sensor on the root arm. (a) Overview. (b) Top view. (c) Bottom view.
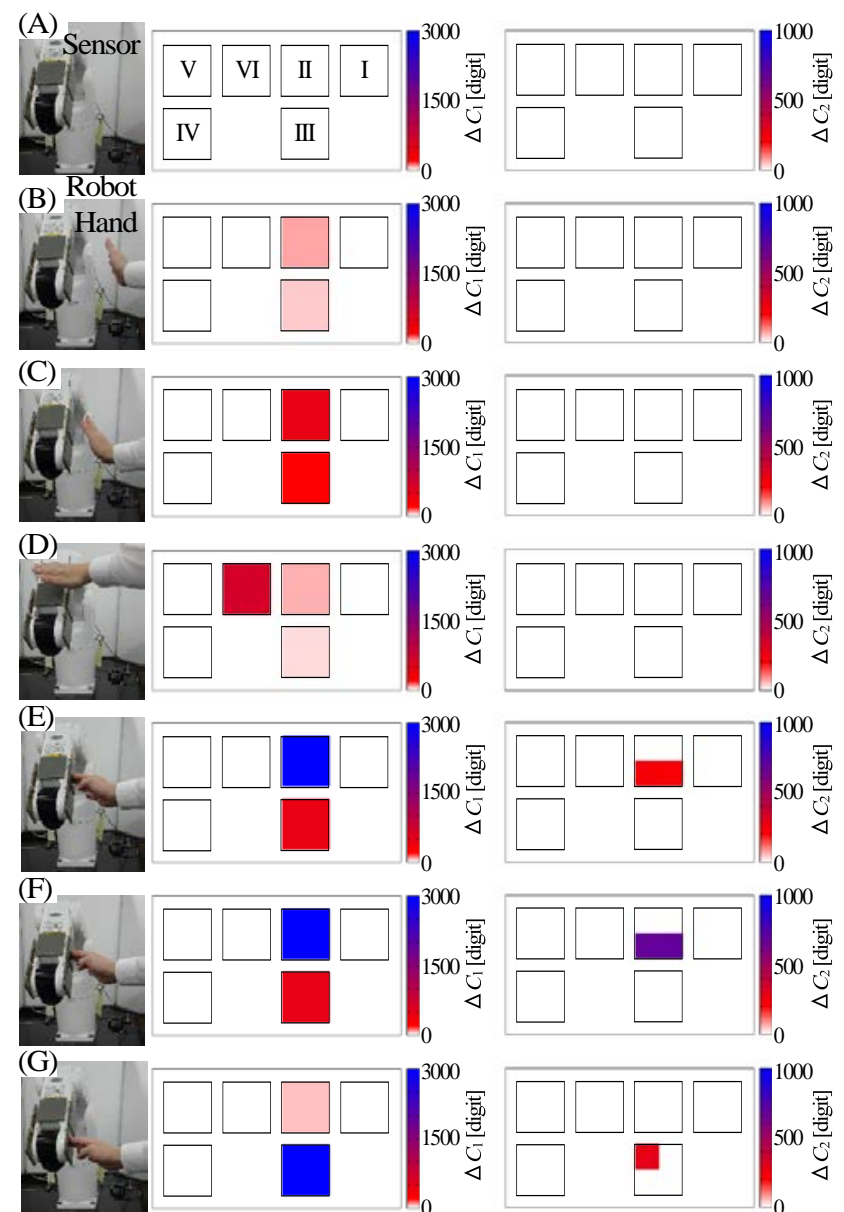

(a)

(b)

(c)

Fig. 9. Measurement results. (a) The position between the sensor and the object. (b) $\Delta C_{1}$. (c) $\Delta C_{2}$.

1000 digits. In Fig. 9(b), $\Delta C_{2}$ changes according to the hand position at non-contact points (B to D). Thus, the sensor could detect the object at proximity range. In Fig. 9 (c), $\Delta C_{1}$ did not change without contact (B to D). In addition, $\Delta C_{1}$ changes according to the pressure and its position when the object was pushed (E to $G$ ). Thus, the sensor could detect the pressure and its position.

The SD of $\Delta C_{2}$ of sensor I on the robot arm was 2.8 
digits when the object far and the robot motor power off. On the other hand, SD of $\Delta C_{2}$ of sensor I on the robot arm was 11.1 digits when the object far and the robot motor power on. The noise increased from the robot arm. However, the sensor could detect the object (human) within approximately $100 \mathrm{~mm}$ when the object is grounded conductor (Hand). Thus, we think that the robot can avoid unnecessary contact with humans ${ }^{(11)}$.

\section{Conclusions}

In this paper, we proposed a tactile and proximity sensor module using a self-capacitance measurement for a HCR. An advantage of this sensor module is that the sensor is easy to add because an $\mathrm{I}^{2} \mathrm{C}$ bus and a slave microcomputer is used for communication between sensors. The proposed sensor consists of two electrodes $\left(E_{1}\right.$ and $\left.E_{2}\right)$ and an elastic body. We placed six sensor modules on the one $\mathrm{I}^{2} \mathrm{C}$ bus line to cover the robot surface. The prototype sensors detected the object (human) at proximity range, and discriminated the material upon contact. Moreover, the sensors detected the pressure and the object position upon contact. The sensors were attached to the robot arm surface and detected objects both before and after contact. The proposed sensor modules can thus be used as tactile sensors for HCRs.

\section{Acknowledgment}

This work was supported by JSPS KAKENHI Grant Number 19K14952.

\section{References}

(1) A. Cirillo, F. Ficuciello, C. Natale, S. Pirozzi, and L. Villani, A Conformable Force/Tactile Skin for Physical Human-Robot Interaction”, IEEE Robotics and Automation Letters, Vol. 1, No. 1, pp. 41-48, 2016

(2) M. Shimojo, T. Araki, A. Ming, and M. Ishikawa, "A High-Speed Mesh of Tactile Sensors Fitting Arbitrary Surfaces”, IEEE Sensors Journal, Vol. 10, No. 4, pp. 822-830, 2010

(3) P. Maiolino, M. Maggiali, G. Cannata, G. Metta, and L. Natale, "A Flexible and Robust Large Scale Capacitive Tactile System for Robots”, IEEE Sensors Journal, Vol.13, No.10, pp. 3910-3917, 2013

(4) KUKA LBR iiwa, https://www.robots.com/series/kukacollaborative-robot-series, access date: May 25, 2019
(5) H. Hasegawa, Y. Suzuki, A. Ming, K. Koyama, M. Ishikawa, and M. Shimojo, "Net-Structure Proximity Sensor: High-Speed and Free-Form Sensor With Analog Computing Circuit”, IEEE/ASME Transactions on Mechatronics, Vol. 20, No. 6, pp. 3232-3241, 2015

(6) T. Schlegl, T. Kroger, A. Gaschler, O. Khatib, and H. Zangl, "Virtual Whiskers-Highly Responsive Robot Collision Avoidance", Proceedings of IEEE/RSJ International Conference on Intelligent Robots and Systems, 2013, pp. 5373-5379

(7) BOSCH APAS, https://www.bosch-apas.com/en/home/, access date: May 25, 2019

(8) D. Hughes, J. Lammie, and N. Correll, "A Robotic Skin for Collision Avoidance and Affective Touch Recognition”, IEEE Robotics and Automation Letters, Vol. 3, No. 3, pp. 1386-1393, 2018

(9) H. Lee, S. Chang, and E. Yoon, "Dual-Mode Capacitive Proximity Sensor for Robot Application: Implementation of Tactile and Proximity Sensing Capability on a Single Polymer Platform Using Shared Electrodes”, IEEE Sensors Journal, Vol. 9, No. 12, pp. 1748-1755, 2009

(10)D. Goger, H. Alagi, and H.Worn, "Tactile proximity sensors for robotic applications”, Proceedings of IEEE International Conference on Industrial Technology, 2013, pp. 978-983

(11) S. Tsuji and T. Kohama, "Proximity and Tactile Sensor Using Self-Capacitance Measurement for Human Collaboration Robots”, IEEJ Transactions on Sensors and Micromachines, Vol. 138, No. 1, pp. 2-8, 2018

(12) S. Tsuji and T. Kohama, "Operating Method of Human Collaborative Robot using Self-Capacitance Proximity and Tactile Sensor", Journal of the Institute of Industrial Applications Engineers Vol. 7, No. 1, pp. 915, 2019 